\title{
PERCEPÇÃO DA IMAGEM CORPORAL ASSOCIADA AO ESTADO NUTRICIONAL DE CRIANÇAS E ADOLESCENTES
}

\section{Perception of body image associated with the nutritional status of children and adolescents \\ Percepción de la imagen corporal asociada con el estado nutricional de niños y adolescentes}

\author{
Maiara Battisti \\ Centro Universitário Univates - Lajeado (RS) - Brasil \\ Patrícia Bergjohann \\ Centro Universitário Univates - Lajeado (RS) - Brasil \\ Fernanda Scherer Adami \\ Centro Universitário Univates - Lajeado (RS) - Brasil \\ Patricia Fassina \\ Centro Universitário Univates - Lajeado (RS) - Brasil
}

\section{RESUMO}

Objetivo: Analisar a relação entre a percepção dos pais quanto à imagem corporal de seus filhos e a autopercepção da imagem corporal das crianças e dos adolescentes em relação ao seu estado nutricional. Métodos: Estudo de caráter transversal, realizado entre março e abril de 2016 com 122 crianças e adolescentes, de 6 a 19 anos, e com os seus responsáveis. Com os escolares, foi aplicado um questionário contendo dados relativos ao gênero, peso, altura e à localização da área da escola (rural ou urbana), além da escala de silhuetas de Stunkard para avaliação da autopercepção da imagem corporal. Calcula-se o estado nutricional pelo índice de massa corporal (IMC) classificando-o de acordo com os pontos de corte da Organização Mundial da Saúde (OMS) 2007. Com os pais ou responsáveis, utilizou-se a escala de silhuetas de Stunkard e uma questão fechada sobre a preocupação com o estado nutricional da criança ou adolescente, além da identificação do gênero, idade e grau de parentesco (pais, avós, tios, irmão ou outro familiar). Dados analisados com teste de Fisher e p $\leq 0,05$ Resultados: Verificouse que a percepção da imagem corporal dos pais esteve significativamente associada à autopercepção da imagem corporal de seus filhos $(p \leq 0,001)$, bem como a autopercepção da imagem corporal dos escolares esteve associada ao seu estado nutricional ( $\mathrm{p} \leq 0,001)$. Conclusão: Houve uma associação positiva entre a percepção dos pais em relação à imagem corporal de seus filhos e entre a autopercepção da imagem corporal das crianças e dos adolescentes com o seu estado nutricional.

Descritores: Imagem Corporal; Estado Nutricional; Criança; Adolescente.

\section{ABSTRACT}

Objective: To analyze the association between the parents' perception of their children's body image and the self-perceived body image of children and adolescents in relation to their nutritional status. Methods: Cross-sectional study conducted between March and April 2016 with 122 children and adolescents aged 6 to 19 years and their guardians. With the students, a questionnaire with data addressing gender, weight, height and school location (rural or urban) was applied, in addition to the Stunkard's Figure Rating Scale (1983) for evaluation of the self-perceived body image. The nutritional status was calculated by means of the Body Mass Index and classified according to the World Health Organization (WHO) 2007 cut-off points. With the parents or guardians, the Stunkard's Figure Rating Scale (1983) and a closed question addressing the concern about their children's nutritional status were used, besides the identification of gender, age and degree of kinship (parents, grandparents, uncles, brother or other family member). Data was analyzed using Fisher's test and $p \leq 0.05$. Results: It was found that the parent's perception of their children's body image was significantly associated with their children's self-perceived body image ( $p \leq 0.001)$, and the students'self-perceived body image was also associated with their nutritional status ( $\leq \leq 0.001)$. Conclusion: There was a positive association between the parent's perception of their children's body image and between the self-perceived body image of children and adolescents and their nutritional status.

Descriptors: Body Image; Nutritional Status; Child; Adolescent. 


\section{RESUMEN}

Objetivo: Analizar la relación entre la percepción de los padres respecto la imagen corporal de sus hijos y la autopercepción de la imagen corporal de niños y adolescentes sobre su estado nutricional. Métodos: Estudio de carácter transversal realizado entre marzo y abril de 2016 con 122 niños y adolescentes entre 6 y 19 años y sus responsables. Se aplicó un cuestionario a los escolares con datos sobre el género, el peso, la altura y la localización del área de la escuela (rural o urbana) además de la escala de siluetas de Stunkard para la evaluación de la autopercepción de la imagen corporal. Se calculó el estado nutricional por el Índice de Masa Corporal (IMC) clasificado según los puntos de corte de la Organización Mundial de la Salud (OMS) de 2007. Se aplicó la escala de siluetas de Stunkard a los padres o responsables y una pregunta cerrada sobre la preocupación del estado nutricional del niño o adolescente, además de la identificación del género, la edad y el vínculo familiar (padres, abuelos, tíos, hermano u otro familiar). Los datos fueron analizados con la prueba de Fisher y p $\leq 0,05$. Resultados: Se verificó que la percepción de la imagen corporal de los padres se asoció de modo significativo con la autopercepción de la imagen corporal de sus hijos $(p \leq 0,001)$ así como la autopercepción de la imagen corporal de los escolares se asoció con su estado nutricional ( $p \leq 0,001)$. Conclusión: Hubo una asociación positiva entre la percepción de los padres sobre la imagen corporal de sus hijos y entre la autopercepción de la imagen corporal de los niños y de los adolescentes con su estado nutricional.

Descriptores: Imagen Corporal; Estado Nutricional; Niño; Adolescente.

\section{INTRODUÇÃO}

A imagem corporal (IC) tem sua relevância por influenciar na construção da identidade do indivíduo, na percepção que o mesmo tem de seu próprio corpo ou do que ele entende como saudável ${ }^{(1)}$. A insatisfação da IC das crianças e dos adolescentes em relação à forma física torna-se mais intensa ao longo do tempo, de forma que tentam mudar sua aparência devido à dificuldade de aceitar o seu corpo ${ }^{(2)}$.

Nesse sentido, a baixa autoestima predomina, aumentando as chances do desenvolvimento de comportamentos de risco, como um transtorno alimentar (TA) $)^{(3)}$, e também patologias, como a obesidade, o diabetes mellitus e a hipertensão arterial ${ }^{(2)}$. Os TAs ocorrem por consequência da percepção distorcida da autoimagem na proporção de que indivíduos eutróficos se veem magros e indivíduos com excesso de peso não se enxergam nessa condição, sendo que os TAs são mais frequentes no gênero feminino e durante a adolescência ${ }^{(4)}$. Assim, a insatisfação com a IC determina a suscetibilidade de desenvolvimento de distúrbios de conduta alimentar ${ }^{(5)}$.

No que diz respeito aos índices elevados de doenças crônicas e à obesidade nesta faixa etária, é possível inferir que a obesidade vem sendo considerada uma epidemia inserida na condição de um fenômeno global, principalmente em países desenvolvidos e industrializados ${ }^{(4,5)}$.

Os distúrbios nutricionais e as patologias associadas à IC podem estar relacionados aos hábitos das crianças e dos adolescentes, que apresentam uma alimentação com alto índice de calorias e não são incentivados à prática de exercícios físicos, permanecendo a maior parte do tempo em contato com meios tecnológicos e eletrônicos ${ }^{(6)}$. Além disso, o grau de instrução e a renda possuem influência na escolha dos alimentos que, na atualidade, devido à praticidade, ao custo e ao marketing, acaba sendo por refrigerantes, sucos industrializados, salgados industrializados e frituras ${ }^{(5)}$, em detrimento de alimentos mais saudáveis para contemplar uma refeição adequada e balanceada, especialmente necessária nesse ciclo de vida devido ao crescimento e ao desenvolvimento $^{(7)}$.

Considera-se que a frequente alimentação deficiente, em termos qualitativos, influencie na percepção da IC que inicia na adolescência, favorecida pelas questões psicológicas, emocionais, somáticas e cognitivas devido à preocupação e à busca por um corpo bonito e boa forma corporal, aspectos atrelados ao ideal imposto pela sociedade ${ }^{(8)}$. A adoção de hábitos adequados, nessa fase da vida, considerando as possíveis consequências de uma alimentação desequilibrada, como a insatisfação com o corpo, deve estar entre a preocupação dos responsáveis, tanto no ambiente doméstico quanto escolar ${ }^{(9)}$.

A percepção dos pais quanto à IC de seus filhos deve representar reflexões constantes sobre esse acompanhamento. Porém, o tempo reduzido para comprar e para preparar alimentos saudáveis, bem como para executar atividades relacionadas à saúde e forma da IC, acaba impactando na alimentação de crianças e adolescentes ${ }^{(10)}$.

Diante do exposto, o objetivo do presente estudo foi analisar a relação entre a percepção dos pais quanto à imagem corporal de seus filhos e a autopercepção da imagem corporal das crianças e dos adolescentes em relação ao seu estado nutricional.

\section{MÉTODOS}

Estudo de caráter transversal, realizado entre março e abril de 2016 com crianças e adolescentes de 6 a 19 anos incompletos, de ambos os gêneros, matriculados nas escolas municipais de uma cidade do interior do estado do Rio Grande do Sul (RS), bem como os pais ou responsáveis pelas crianças e adolescentes. No município do presente estudo, a rede municipal era composta por seis escolas de ensino fundamental que, juntas, somavam um total de 331 escolares que atenderam aos critérios de inclusão. 
Os(as) diretores(as) das escolas e os pais ou responsáveis dos alunos, inclusive os próprios escolares, receberam o convite para participar do estudo e, após expressarem concordância verbal, receberam o TCLE.

Participaram do estudo 122 crianças e adolescentes que compuseram a amostra final, sendo que os 209 faltantes não devolveram o TCLE assinado pelos pais ou responsáveis, entendendo-se como uma recusa em participar do estudo. Foram excluídos os escolares, e consequentemente os pais ou responsáveis que não estiveram presentes na data da coleta de dados, que desistiram do estudo por algum motivo, que estiveram impossibilitados de realizar a avaliação nutricional ou de responder aos questionários, bem como aqueles que não assinaram o Termo de Consentimento Livre e Esclarecido (TCLE).

A coleta de dados consistiu, no caso dos pais ou responsáveis, na aplicação de um questionário estruturado sobre a percepção do estado nutricional, descrito pela escala de silhuetas de Stunkard ${ }^{(11)}$, contendo uma questão fechada sobre a preocupação do estado nutricional do seu filho e adaptada utilizando a classificação "nada preocupado", "preocupado" e "muito preocupado"(12). Além disso, realizou-se a identificação do gênero, idade e grau de parentesco (pais, avós, tios, irmão ou outro familiar).

A escala de silhuetas de Stunkard ${ }^{(1)}$, contida no questionário para a percepção da IC dos pais ou responsáveis em relação ao estado nutricional de seus filhos, considerou imagens de 1 a 9 , de meninos e meninas, com silhuetas que abrangem desde a magreza até a obesidade, sendo a silhuetas 1 considerada baixo peso; as silhuetas 2 e 3 , normais; as silhuetas de 4 a 6 , sobrepeso, e as silhuetas de 7 a 9 , obesidade. Nessa escala, os pais ou responsáveis assinalaram com um "x" qual imagem melhor se parecia com a de seu (sua) filho(a).

Aplicou-se a mesma escala de silhuetas(11) para a autopercepção da IC, utilizando os mesmos critérios de classificação para as crianças e os adolescentes, bem como a realização da avaliação antropométrica, que consistiu na verificação do peso e altura para posterior avaliação do estado nutricional.

Verificou-se o peso com uma balança digital calibrada, da marca Cadence $^{\circledR}$, com capacidade para 150 quilogramas e precisão de 100 gramas, estando o participante com o mínimo de indumentária e sem o uso de calçados, posicionado no centro do equipamento, ereto, com os pés juntos e os braços estendidos ao longo do corpo, segundo as normas preconizadas pelo Sistema de Vigilância Alimentar e Nutricional (SISVAN) ${ }^{(13)}$.

Para aferição da altura, utilizou-se uma fita métrica, posicionando o participante com a cabeça livre de adereços, em pé, ereto, com os braços estendidos ao longo do corpo e a cabeça erguida, olhando para um ponto fixo na altura dos olhos, no plano de Frankfurt, segundo as normas preconizadas pelo SISVAN ${ }^{(13)}$.

Para avaliação do estado nutricional, foi utilizado o cálculo do índice de massa corporal (IMC) que considera a relação entre o peso e a altura ao quadrado (IMC $=$ Peso em $\mathrm{kg}$ /Altura $\mathrm{em} \mathrm{m}^{2}$ ). Realizou-se a classificação do IMC de acordo com os pontos de corte da Organização Mundial da Saúde (OMS) ${ }^{(14)}$ para faixa etária e gênero. Para otimizar a avaliação do estado nutricional dos escolares, optou-se por agrupar essas categorias de classificação do IMC optando-se por magreza ( $<$ Percentil 3); eutrofia ( $\geq$ Percentil 3 e $\leq$ Percentil 85); sobrepeso ( $>$ Percentil 85 e $\leq$ Percentil 97) e obesidade ( $>$ Percentil 97).

Os dados foram analisados através de tabelas, de estatísticas descritivas e do Teste Exato de Fisher, para o qual assumiu-se o nível de significância máximo de 5\% ( $\leq \leq 0,05)$ e utilizou-se o software Statistical Package for the Social Sciences (SPSS), versão 13.0, para a análise estatística.

O presente estudo recebeu aprovação do Comitê de Ética em Pesquisa do Centro Universitário Univates, pelo protocolo de número 612.030 .

\section{RESULTADOS}

A média de idade foi de 10,32 $\pm 2,39$ anos, sendo, portanto, a maioria crianças, $63,1 \%(\mathrm{n}=77)$; com 8 anos, 17,2\% ( $\mathrm{n}=21)$; e pertencentes ao sexo feminino, 65,6\% $(\mathrm{n}=80)$. Quanto à localização da escola, a maioria, $52,5 \%(\mathrm{n}=64)$, era estudante de escolas localizadas na zona urbana do município. Segundo a classificação do IMC, a maioria, 73,0\% (n=89), apresentou eutrofia, com média de IMC de 18,16 $\pm 3,63 \mathrm{~kg} / \mathrm{m}^{2}$. As médias de peso e altura foram de 39,48 $\pm 13,2$ quilos e de 1,45 $\pm 0,14$ metros respectivamente. Em relação ao grau de parentesco dos responsáveis com os escolares que participaram do estudo, $95,1 \%(n=116)$ eram pais (pai ou mãe) (Tabela I).

Sobre a autopercepção da IC conforme a escala de silhuetas, $50 \%(\mathrm{n}=61)$ dos escolares se caracterizaram como eutróficos e $37,7 \%(\mathrm{n}=46)$ com excesso de peso. Quanto à preocupação do responsável em relação ao estado nutricional de seu filho, 45,9\% $(n=56)$ deles demonstraram estar "preocupado", enquanto 43,4\% (n=53) apresentaram-se como "nada preocupado". Em relação à percepção da IC pelos pais em relação aos filhos, conforme a escala de silhuetas, 49,2\% $(n=60)$ relacionou eutrofia, enquanto $36,1 \%(n=44)$, excesso de peso (Tabela I). 
Tabela I - Caracterização da população estudada. Rio Grande do Sul, 2016.

\begin{tabular}{|c|c|c|}
\hline Variável & $\mathbf{n}$ & $\%$ \\
\hline \multicolumn{3}{|l|}{ Sexo } \\
\hline Masculino & 42 & 34,4 \\
\hline Feminino & 80 & 65,6 \\
\hline \multicolumn{3}{|l|}{ Idade (anos) } \\
\hline 6 & 5 & 4,1 \\
\hline 7 & 11 & 9 \\
\hline 8 & 21 & 17,2 \\
\hline 9 & 11 & 9 \\
\hline 10 & 15 & 12,3 \\
\hline 11 & 14 & 11,5 \\
\hline 12 & 19 & 15,6 \\
\hline 13 & 11 & 9 \\
\hline 14 & 15 & 12,3 \\
\hline \multicolumn{3}{|l|}{ Localização da escola } \\
\hline Urbana & 64 & 52,5 \\
\hline Rural & 58 & 47,5 \\
\hline \multicolumn{3}{|l|}{ Grau de parentesco } \\
\hline Pai/Mãe & 116 & 95,1 \\
\hline Avô/Avó & 5 & 4,1 \\
\hline Tio/Tia & 1 & 0,8 \\
\hline \multicolumn{3}{|c|}{ IMC - Classificação agrupada } \\
\hline Magreza & 6 & 4,9 \\
\hline Eutrofia & 89 & 73 \\
\hline Sobrepeso & 17 & 13,9 \\
\hline Obesidade & 10 & 8,2 \\
\hline \multicolumn{3}{|c|}{ Autopercepção - Escala de silhuetas } \\
\hline Baixo peso & 15 & 12,3 \\
\hline Normal & 61 & 50 \\
\hline Sobrepeso & 34 & 27,9 \\
\hline Obesidade & 12 & 9,8 \\
\hline \multicolumn{3}{|c|}{ Preocupação do responsável } \\
\hline Nada preocupado & 53 & 43,4 \\
\hline Preocupado & 56 & 45,9 \\
\hline Muito preocupado & 13 & 10,7 \\
\hline \multicolumn{3}{|l|}{ Percepção da IC } \\
\hline Baixo peso & 18 & 14,8 \\
\hline Normal & 60 & 49,2 \\
\hline Sobrepeso & 34 & 27,9 \\
\hline Obesidade & 10 & 8,2 \\
\hline
\end{tabular}

IMC: Índice de Massa Corporal. IC: Imagem Corporal.

A percepção da IC pelos pais, em relação ao estado nutricional de seus filhos, apontou uma percepção com significativa concordância com a classificação do estado nutricional de seu filho, incluindo todas as categorias agrupadas de IMC ( $\mathrm{p} \leq 0,001)$. Assim, a percepção de baixo peso dos pais esteve associada à classificação IMC de magreza do filho em $66,7 \%(\mathrm{n}=4)$ dos casos. A percepção normal esteve associada à classificação eutrofia em 62,9\% $(n=56)$. Por sua vez, a percepção de sobrepeso associouse à classificação IMC sobrepeso em 76,5\% $(n=13)$. Por fim, a percepção obesidade mostrou-se associada à classificação IMC obesidade em $60,0 \%(n=6)$ (Tabela II).

Tabela II - Percepção da imagem corporal dos pais em relação ao estado nutricional de seus filhos, segundo classificação agrupada pelo índice de massa corporal (IMC). Rio Grande do Sul, 2016.

\begin{tabular}{|c|c|c|c|c|c|c|c|c|c|}
\hline \multirow{3}{*}{ Variável } & \multirow{3}{*}{ Resposta } & \multicolumn{8}{|c|}{ IMC classificação agrupada } \\
\hline & & \multicolumn{2}{|c|}{ Magreza } & \multicolumn{2}{|c|}{ Eutrofia } & \multicolumn{2}{|c|}{ Sobrepeso } & \multicolumn{2}{|c|}{ Obesidade } \\
\hline & & $\mathbf{n}$ & $\%$ & n & $\%$ & $\mathbf{n}$ & $\%$ & n & $\%$ \\
\hline \multirow{4}{*}{$\begin{array}{l}\text { Percepção da IC dos pais em } \\
\text { relação aos filhos conforme } \\
\text { escala de silhuetas }\end{array}$} & Baixo Peso & $4 *$ & $66,7^{*}$ & 13 & 14,6 & 1 & 5,9 & - & - \\
\hline & Normal & 2 & 33,3 & $56^{*}$ & $62,9 *$ & 2 & 11,8 & - & - \\
\hline & Sobrepeso & - & - & 17 & 19,1 & $13 *$ & $76,5^{*}$ & 4 & 40,0 \\
\hline & Obesidade & - & - & 3 & 3,4 & 1 & 5,9 & $6^{*}$ & $60,0 *$ \\
\hline
\end{tabular}

IMC: Índice de Massa Corporal; IC: Imagem Corporal. Teste Exato de Fisher. *significativo p $\leq 0,05$ 
Verificou-se que a autopercepção da imagem corporal dos escolares esteve associada de modo significativo ao seu estado nutricional $(\mathrm{p} \leq 0,001)$, sendo que a autopercepção normal foi associada à classificação de eutrofia em $61,8 \%(\mathrm{n}=55)$ dos casos. A autopercepção de sobrepeso esteve associada às classificações de IMC sobrepeso e obesidade em $58,8 \%(\mathrm{n}=10)$ e $60,0 \%$ $(\mathrm{n}=6)$ respectivamente; e a autopercepção obesidade mostrou associação com as classificações de IMC sobrepeso e obesidade em $23,5 \%(n=4)$ e 40,0\% $(n=4)$ respectivamente $(p \leq 0,001)$. Portanto, somente a autopercepção baixo peso não se apresentou associada de modo significativo a nenhuma classificação de IMC (Tabela III).

Tabela III - Autopercepção da Imagem Corporal dos escolares em relação ao seu estado nutricional, segundo classificação agrupada pelo índice de massa corporal (IMC). Rio Grande do Sul, 2016.

\begin{tabular}{|c|c|c|c|c|c|c|c|c|c|}
\hline \multirow{3}{*}{ Variável } & \multirow{3}{*}{ Resposta } & \multicolumn{8}{|c|}{ IMC classificação agrupada } \\
\hline & & \multicolumn{2}{|c|}{ Magreza } & \multicolumn{2}{|c|}{ Eutrofia } & \multicolumn{2}{|c|}{ Sobrepeso } & \multicolumn{2}{|c|}{ Obesidade } \\
\hline & & $\mathbf{n}$ & $\%$ & $\mathbf{n}$ & $\%$ & $\mathbf{n}$ & $\%$ & n & $\%$ \\
\hline \multirow{4}{*}{$\begin{array}{l}\text { Autopercepção da IC conforme a } \\
\text { escala de silhuetas }\end{array}$} & Baixo Peso & 1 & 16,7 & 13 & 14,6 & 1 & 5,9 & - & - \\
\hline & Normal & 4 & 66,7 & 55 & $61,8^{*}$ & 2 & 11,8 & - & - \\
\hline & Sobrepeso & 1 & 16,7 & 17 & 19,1 & 10 & $58,8 *$ & 6 & $60,0^{*}$ \\
\hline & Obesidade & - & - & 4 & 4,5 & 4 & $23,5^{*}$ & 4 & $40,0^{*}$ \\
\hline
\end{tabular}

IMC: Índice de Massa Corporal; IC: Imagem Corporal. Teste Exato de Fisher. *significativo p $\leq 0,05$

Observou-se que a percepção da IC dos pais em relação à autopercepção da IC de seus filhos esteve significativamente associada $(\mathrm{p} \leq 0,001)$, ou seja, a percepção baixo peso dos pais esteve associada à autopercepção baixo peso dos filhos em $80,0 \%$ $(\mathrm{n}=12)$ dos casos. A percepção normal dos pais apresentou-se associada à autopercepção normal dos filhos em 82,0\% ( $\mathrm{n}=50)$; $\mathrm{o}$ mesmo ocorreu com a percepção de sobrepeso e obesidade dos pais, que se mostrou relacionada às autopercepções sobrepeso e obesidade dos filhos em 58,8\% $(n=20), 66,7 \%(n=8), 17,6 \%(n=6)$ e 33,3\% $(n=4)$ respectivamente (Tabela IV).

Tabela IV - Percepção da imagem corporal dos pais em relação à autopercepção da imagem corporal de seus filhos. Rio Grande do Sul, 2016.

\begin{tabular}{|c|c|c|c|c|c|c|c|c|c|}
\hline \multirow{3}{*}{ Variável } & \multirow{3}{*}{ Resposta } & \multicolumn{8}{|c|}{ Autopercepção da imagem corporal } \\
\hline & & \multicolumn{2}{|c|}{ Baixo Peso } & \multicolumn{2}{|c|}{ Normal } & \multicolumn{2}{|c|}{ Sobrepeso } & \multicolumn{2}{|c|}{ Obesidade } \\
\hline & & n & $\%$ & n & $\%$ & n & $\%$ & $\mathbf{n}$ & $\%$ \\
\hline \multirow{4}{*}{$\begin{array}{l}\text { Percepção da IC dos pais em relação } \\
\text { aos filhos conforme escala de } \\
\text { silhuetas }\end{array}$} & Baixo Peso & $12^{*}$ & $80,0^{*}$ & 5 & 8,2 & 1 & 2,9 & - & - \\
\hline & Normal & 3 & 20,0 & $50 *$ & $82,0 *$ & 7 & 20,6 & - & - \\
\hline & Sobrepeso & - & - & 6 & 9,8 & $20 *$ & $58,8^{*}$ & $8^{*}$ & $66,7^{*}$ \\
\hline & Obesidade & - & - & - & - & $6^{*}$ & $17,6^{*}$ & $4^{*}$ & $33,3 *$ \\
\hline
\end{tabular}

IC: Imagem Corporal. Teste Exato de Fisher. *significativo p $\leq 0,05$

Não se constatou associação significativa entre a percepção da IC e o estado nutricional dos escolares em relação à zona de localização da escola (Tabela V).

Tabela V - Associação entre a percepção da imagem corporal dos pais em relação aos filhos e a classificação agrupada do IMC em relação à zona de localização da escola. Rio Grande do Sul, 2016.

\begin{tabular}{|c|c|c|c|c|c|c|}
\hline \multirow{3}{*}{ Variável } & \multirow{3}{*}{ Resposta } & \multicolumn{4}{|c|}{ Localização da escola } & \multirow{3}{*}{ p-valor } \\
\hline & & \multicolumn{2}{|c|}{ Urbana } & \multicolumn{2}{|c|}{ Rural } & \\
\hline & & $\mathbf{n}$ & $\%$ & $\mathbf{n}$ & $\%$ & \\
\hline \multirow{3}{*}{ IMC classificação agrupada } & Magreza & 4 & 6,3 & 2 & 3,4 & 0,813 \\
\hline & Eutrofia & 45 & 70,3 & 44 & 75,9 & \\
\hline & Sobrepeso & 9 & 14,1 & 8 & 13,8 & \\
\hline \multirow{5}{*}{ Percepção da IC dos pais em relação aos filhos } & Obesidade & 6 & 9,4 & 4 & 6,9 & \\
\hline & Baixo Peso & 8 & 12,5 & 10 & 17,2 & 0,890 \\
\hline & Normal & 32 & 50,0 & 28 & 48,3 & \\
\hline & Sobrepeso & 18 & 28,1 & 16 & 27,6 & \\
\hline & Obesidade & 6 & 9,4 & 4 & 6,9 & \\
\hline
\end{tabular}

IMC: Índice de Massa Corporal; IC: Imagem Corporal. Teste Exato de Fisher. *significativo p $\leq 0,05$. 


\section{DISCUSSÃO}

Os resultados evidenciados no presente estudo indicam uma relação positiva com a classificação do estado nutricional entre a autopercepção da IC das crianças/ adolescentes e a dos pais em relação aos seus filhos. Contudo, é de fundamental importância que os profissionais de saúde abordem o assunto da relação entre a IC e o estado nutricional com as crianças e os adolescentes com vistas à prevenção de doenças e à promoção da saúde.

Sobre a percepção da IC dos pais em relação ao estado nutricional de seus filhos, o presente estudo observou que a percepção dos pais esteve em concordância com a classificação do estado nutricional de seu filho para todas as categorias agrupadas de IMC. Um estudo ${ }^{(15)}$ sobre a percepção da IC materna em relação ao estado nutricional dos filhos, demonstrou que as mães apresentaram a IC distorcida em relação ao excesso de peso, subestimando o estado nutricional de seus filhos, o que corrobora com um outro estudo no qual os pais também tenderam a subestimar o estado nutricional da criança ao não reconheceram a existência de obesidade nos seus filhos, indicando uma distorção da IC de seus filhos ${ }^{(12)}$. Outro estudo apontou que alguns pais têm uma IC distorcida de seus filhos por desejarem perceber os fatos como lhes interessa ${ }^{(16)}$.

No estudo em questão a autopercepção da IC dos escolares esteve associada à realidade do seu estado nutricional, não se constatando distorção, o que corrobora com os resultados de outros estudos que ressaltaram que a maioria dos adolescentes tem uma percepção positiva de sua IC, não apresentando graves distorções ${ }^{(8,17)}$.

Cabe salientar que a IC é a ilustração, tamanho, aparência e forma corporal, sendo que as respostas emocionais são associadas ao estado nutricional e, consequentemente, à satisfação ou insatisfação da $\mathrm{IC}^{(18)}$. Desde a infância, e ainda na adolescência, pode haver distorção da IC, pois a percepção da IC se modifica com o passar do tempo, sendo a imagem que aparece em nossa mente ou que, muitas vezes, queremos ter ${ }^{(17)}$.

O presente estudo apresentou uma associação entre a percepção da IC dos filhos pelos pais associada à autopercepção da IC de seus filhos, como observado em um estudo em que a maioria dos pais percebeu uma IC dos filhos (real) próxima da que gostariam que eles fossem ${ }^{(12)}$. $\mathrm{O}$ atual estudo ainda mostrou que quase metade dos pais ou responsáveis pelos escolares demonstrou preocupação com o estado nutricional de seu filho, e, ao mesmo tempo, quase metade dos pais ou responsáveis pelos escolares apresentaram-se nada preocupados, havendo ainda um percentual menor que se mostrou muito preocupado. Um estudo realizado no Espírito Santo, observou que a maioria das mães referiu preocupação com o peso atual ou futuro de seus filhos ${ }^{(19)}$, assim como outro estudo realizado na cidade de São Carlos, o qual observou uma maior porcentagem de pais preocupados perante os problemas de peso relacionados aos filhos adolescentes ${ }^{(20)}$.

No atual estudo não foi observada associação significativa entre a percepção da IC dos pais em relação aos filhos, nem com o estado nutricional dos escolares em relação à zona de localização da escola. Resultados semelhantes a um estudo realizado nos municípios de Vitória e Santa Maria de Jetibá, onde, tanto na região urbana quanto na rural, a maioria das mães apresentou maiores percentuais de percepção do estado nutricional de seus filhos correspondente ao estado nutricional realmente diagnosticado ${ }^{(19)}$.

Como limitação do estudo, destaca-se a baixa participação por parte dos escolares, no qual mais da metade não demonstrou interesse de participação. Também não houve avaliação da maturidade sexual das crianças e adolescentes, para a qual poderia ter sido aplicada a Escala de Tanner, um instrumento útil na determinação do estágio puberal, que pode estar relacionado ao grau de maturação sexual.

\section{CONCLUSÃO}

O presente estudo apresentou uma associação positiva entre a percepção dos pais em relação à imagem corporal dos filhos e à autopercepção da imagem corporal das crianças e adolescentes com o seu estado nutricional. Assim, os resultados foram positivos, pois foi demonstrado que a percepção dos pais esteve em concordância com a classificação do estado nutricional de seu filho em todas as categorias de índice de massa corporal.

\section{REFERÊNCIAS}

1. Silva MLA, Taquette SR, Coutinho ESF. Sentidos da imagem corporal de adolescentes no ensino fundamental. Rev Saúde Pública. 2014;48(3):438-44.

2. Finatto S, Rech RR, Mignon P, Gavineski IC, Toni V, Halpern R. Insatisfação com a imagem corporal em escolares do sexto ano da rede municipal de Caxias do Sul, no Rio Grande do Sul. Rev Paul Pediatr. 2013;31(1):65-70.

3. Almeida SG. A influência da imagem corporal como causa de transtornos alimentares em adolescentes escolares de uma escola da rede particular de Brasília. Ens Ciênc. 2012; 16(6):107-17.

4. Correia JSC, Falqueto PS, Lopes LM, Gomes SCA. Percepção corporal de adolescentes de uma escola pública de Porto Velho/RO. Ens Ciênc. 2013;17(1):79-88. 
5. Zordão PO, Barbosa A, Parisi TS, Grasselli CSM, Nogueira DA, Silva RR. Associação da imagem corporal e transtornos alimentares em adolescentes de Minas Gerais (Brasil). Nutr Clín Diet Hosp. 2015;35(2):48-56.

6. Ribeiro G, Lopes ERN, Magalhães JC, Andrade MAS. Prevalência de sobrepeso e obesidade em crianças da rede pública de ensino da Cidade de Cruz das Almas, Bahia. Rev Baiana Saúde Pública. 2013;37(1):9-19.

7. Paula FR, Lamboglia CMGF, Silva VTBL, Monteiro MS, Moreira AP, Pinheiro MHNP, et al. Prevalência de sobrepeso e obesidade em escolares da rede pública e particular da cidade de Fortaleza. Rev Bras Promoç Saúde. 2014;27(4):455-61.

8. Gomes MV, Garcia MCR, Franz LBB, Busnello M. Perfil alimentar e antropométrico de crianças e adolescentes praticantes de voleibol. Unijuí: Salão do Conhecimento; 2014.

9. Felden EPG, Claumann GS, Sacomori C, Daronco LSE, Cardoso FL, Pelegrini A. Fatores sociodemográficos e imagem corporal em adolescentes de ensino médio. Ciênc Saúde Coletiva. 2015;20(11):3329-37.

10. Bento IC, Esteves JMM, França TE. Alimentação saudável e dificuldades para torná-la uma realidade: Percepções de pais/ responsáveis por pré-escolares de uma creche em Belo Horizonte/MG, Brasil. Ciênc Saúde Coletiva. 2015;20(8):2389400 .

11. Stunkard AJ, Sorenso T, Schlusinger F. Use of the Danish adoption register for the study of obesity and thinness. Res Publ Assoc Res Nerv Ment Dis. 1983;60:115-20.

12. Aparício G, Cunha M, Duarte J, Pereira A. Olhar dos pais sobre o estado nutricional das crianças pré-escolares. Millenium. 2011;40:99-113.

13. Ministério da Saúde (BR). Orientação para coletas e análise de dados antropométricos em serviço da saúde. Norma técnica do Sistema de Vigilância Alimentar e Nutricional - SISVAN. Brasília: Ministério da Saúde; 2011.

14. World Health Organization - WHO. WHO Growth reference data for 5-19 years, 2007 [acesso em 2016 Maio 31]. Disponível em: http://www.who.int/growthref/en/

15. Friedrich RR, Antunes A, Schuch I. Percepção materna do estado nutricional de crianças em escolas municipais de Porto Alegre - RS. Demetra. 2016;11(1):211-23.

16. Bracht CMB, Piasetzki CTR, Busnello MB, Berlezi EM, Feanz LBB, Boff ETO. Percepção da autoimagem corporal, estado nutricional e prática de atividade física de universitários do Rio Grande do Sul. Mundo Saúde. 2013;37(3):343-53.

17. Florêncio RS, Moreira TMM, Silva MRF, Almeida ILS. Excesso ponderal em adultos jovens escolares: a vulnerabilidade da autopercepção corporal distorcida. Rev Bras Enferm. 2016;69(2):258-65.

18. Gerber KP. Estado nutricional e satisfação com a imagem corporal de trabalhadores ativos em Porto Alegre, RS [trabalho de conclusão de curso]. Porto Alegre: Universidade Federal do Rio Grande do Sul; 2014.

19. Arpini LSB, Barbosa GC, Justo GF, Salaroli BL, Molina MDCB. Associação entre a percepção materna do estado nutricional do filho e a qualidade da dieta de escolares. Demetra. 2015;10(2):299-314.

20. Bello FPS, Chagas NB, Pinto VLM, Camargo LLAL, Demarzo MMP, Germano CMR. Conscientização dos pais sobre o estado nutricional de seus filhos: estudo exploratório em adolescentes de baixa renda no Brasil. Rev Bras Crescimento Desenvolv Hum. 2015;25(3):291-6.

\section{Endereço do primeiro autor:}

Maiara Battisti

Centro Universitário Univates

Rua Avelino Tallini, 171

Bairro: Universitário

CEP: 95900-000 - Lajeado - RS - Brasil

E-mail: maiarab@universo.univates.br

\section{Endereço para correspondência:}

Patricia Fassina

Centro Universitário Univates

Rua Avelino Tallini, 171

Bairro: Universitário

CEP: 95.900-000 - Lajeado - RS - Brasil

E-mail: patriciafassina@univates.br 